\title{
Introduction to crystallography: two-dimensional computer simulations
}

\section{B. D. HAll ${ }^{a}+$ AND T. Stoto ${ }^{b}$ at ${ }^{a}$ Department of Physics, Massey University, Palmerston North, New Zealand, and ${ }^{b}$ The New Zealand Institute for Industrial Research, PO Box 31-310, Lower Hutt, New Zealand. E-mail: b.d.hall@irl.cri.nz}

(Received 19 March 1997; accepted 24 July 1997)

\begin{abstract}
Many elementary concepts of crystallography can be presented in two dimensions, where they are relatively easy to visualize. A computer simulation program for two-dimensional crystallography has been developed for use in teaching at an introductory level. The program will generate and display direct and reciprocal lattices, based on arbitrary user input, and is able to apply the symmetry operations of any of the 17 twodimensional plane groups to a user-defined motif. Examples using the program are presented and discussed.
\end{abstract}

\section{Introduction}

Using two-dimensional crystallography, it is possible to illustrate a variety of concepts relating to crystal symmetry, and to the relationship between crystal structure and the reciprocal lattice (diffraction pattern). Working in two dimensions avoids the difficulty of visualizing three-dimensional structures, making two-dimensional crystallography attractive for teaching at an introductory level.

The purpose of this article is to illustrate how a simple computer program can be used to enhance introductory lessons in crystallography. A number of examples are

† Present address: The New Zealand Institute for Industrial Research, PO Box 31-310, Lower Hutt, New Zealand.

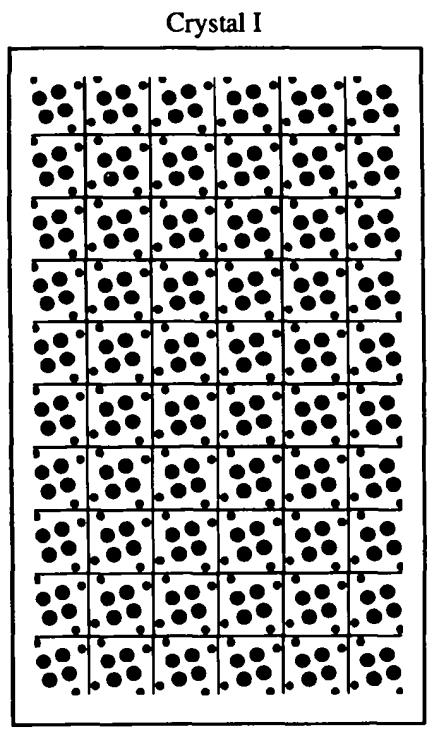

presented below using a simulation program developed for teaching. As far as we know, a similar teaching aid is not currently available. We therefore wish to draw attention to the utility of such a program and make it available to any who wish to try it.

\section{Simple exercises}

The interactive aspect of a computer program provides a direct way for students to hone their understanding of the simple relationships that exist between the direct and reciprocal lattices. For example, a change of scale, or a rotation, are represented on the screen immediately, reinforcing an intuition for these simple transformations.

Another elementary exercise we find useful is for students to investigate the relationship between real and reciprocal lattices starting with a square lattice, then rectangular and finally hexagonal. Again, the exercise aims at building intuition for the reciprocal lattice in general. In going from square to rectangular, the effect of a change of scale in only one dimension is shown; then the hexagonal lattice provides a lattice in which the basis vectors are not orthogonal and hence the reciprocal vectors are not in the same directions as the direct lattice vectors. Most students, in our experience, find their intuition challenged in working through these lattices, especially the hexagonal case. The exercise allows us to focus

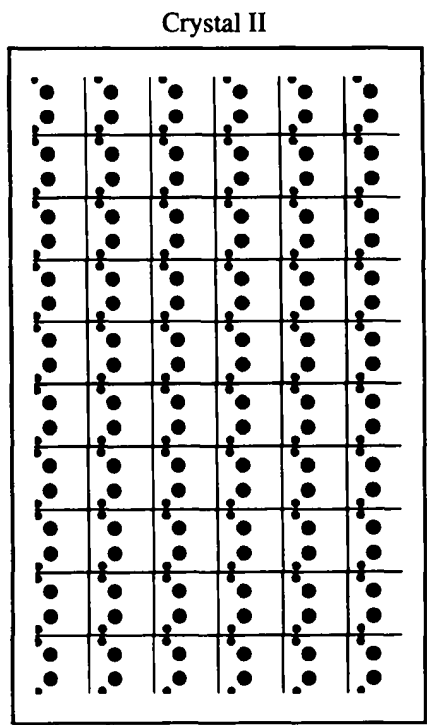

Fig. 1. Two simple crystal structures based on the square lattice, but having distinctly different symmetries: crystal I has $p 4$ symmetry and crystal II has $p 1 \mathrm{~m} 1$ symmetry. Both structures were generated in the program from a two-atom motif to which the symmetry operations of the particular space groups were then applied. 
students' attention on the association of reciprocal-lattice vectors with periodicity in real space.

\section{Lattice and crystal symmetry}

The point symmetry of a lattice and a crystal structure, whose spatial periodicity is defined by that lattice, are not necessarily the same. Symmetry in the crystal may be reduced by decorating the lattice with a pattern of atoms of lower symmetry. This is shown for a square lattice in Fig. 1. The structure on the left (crystal I) has the symmetry of the $p 4$ plane group. The fourfold axes at the center of the square and at the lattice nodes, and the twofold axes midway along the cell edges, are point-symmetry elements that also apply to the square lattice. However, in the figure on the right (crystal II) these elements of symmetry disappear. Here, the plane group is $p 1 m 1$. The crystal structure now has only axes of mirror symmetry running horizontally along the cell edges and through the center of the cell: although the underlying lattice remains square, the structure is now rectangular.

Both figures in this example were generated from the same two-atom motif by applying the appropriate plane-group symmetries to generate the remaining atomic positions. Our program includes the plane-group symmetries, described in the International Tables for Crystallography (Vol. A, 1983), and therefore can be used to develop familiarity with the presen-
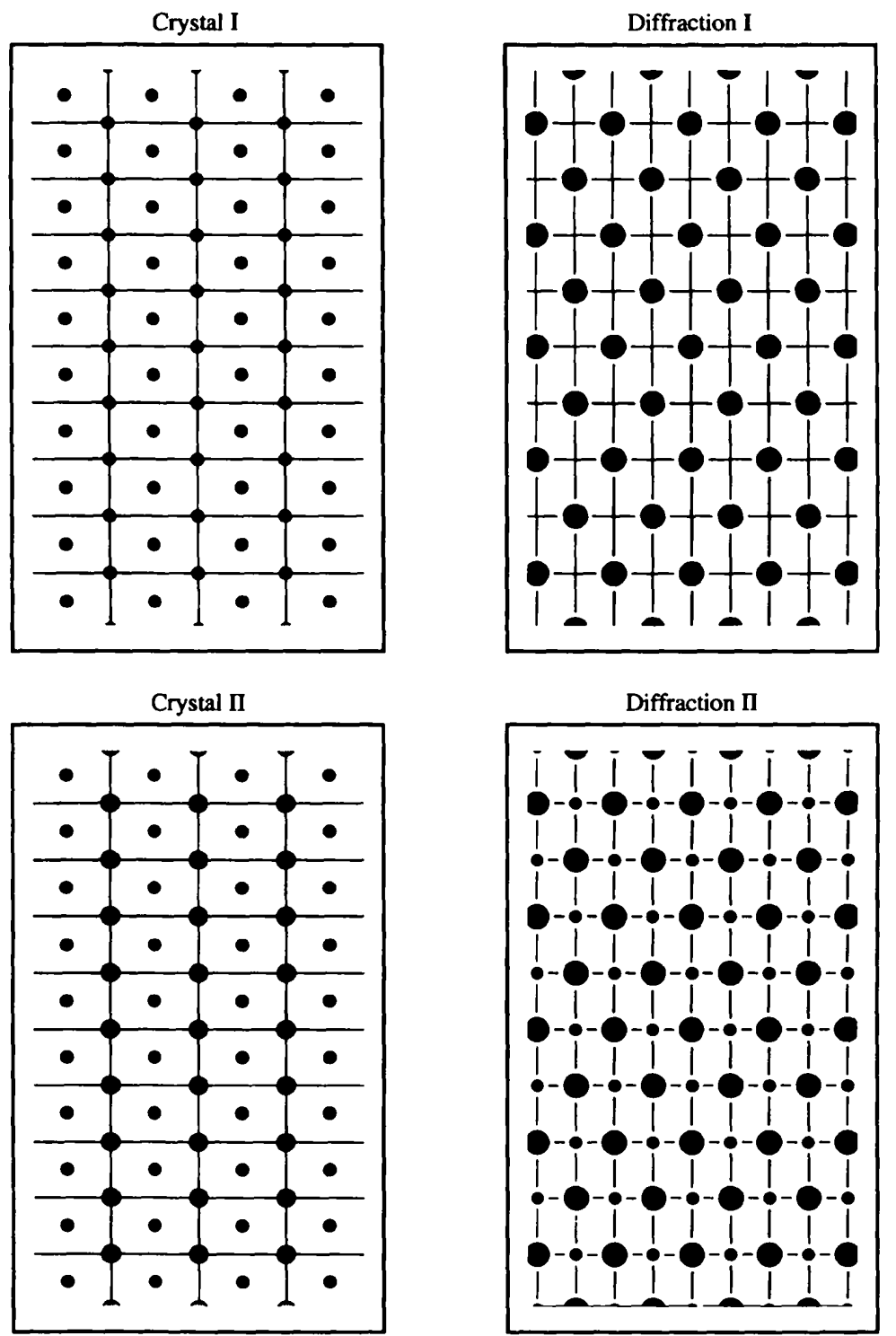

Fig. 2. The upper left panel shows crystal I, a rectangular centered crystal structure, and the panel to the right is the corresponding diffraction pattern. Dark disks in the diffraction pattern represent diffracted intensity (the diameter is proportional to intensity) and lines represent reciprocal-lattice vectors. A systematic absence is indicated by the complete absence of a disk at the intersection of two reciprocal-lattice lines. The two lower panels refer to a structure similar to crystal I, but with different atoms (different form factors) at the center and origin of the unit cell. This changes the cell from centered to primitive, eliminating systematic absences. 
tation of crystallographic data in these tables, as well as to explore the relationships between lattices, point groups and plane groups (Giacovazzo, 1992a). For more advanced students, simple examples of group, subgroup and supergroup relationships can also be developed (Giacovazzo, 1992b; International Tables for Crystallography, 1983).

\section{Conventional and primitive unit cells}

In two dimensions, the only example of a nonprimitive unit cell occurs in the centered rectangular lattice. In this example it is used to illustrate the occurrence of systematic absences in the diffraction pattern.

In Fig. 2, the upper left panel shows a centered rectangular crystal structure (left), with atoms at the center and corners of the unit cell. The corresponding diffraction pattern (Fig. 2, right) shows an absence of scattering intensity at every second node of the reciprocal lattice. The important feature of the diffraction pattern, the regular extinction of intensity, arises from the conventional (rectangular) choice of lattice vectors. The same crystal structure could be generated with different (rhombic) primitive lattice vectors and no symmetry applied (i.e. $p 1)$. In that case, the associated reciprocal-lattice vectors
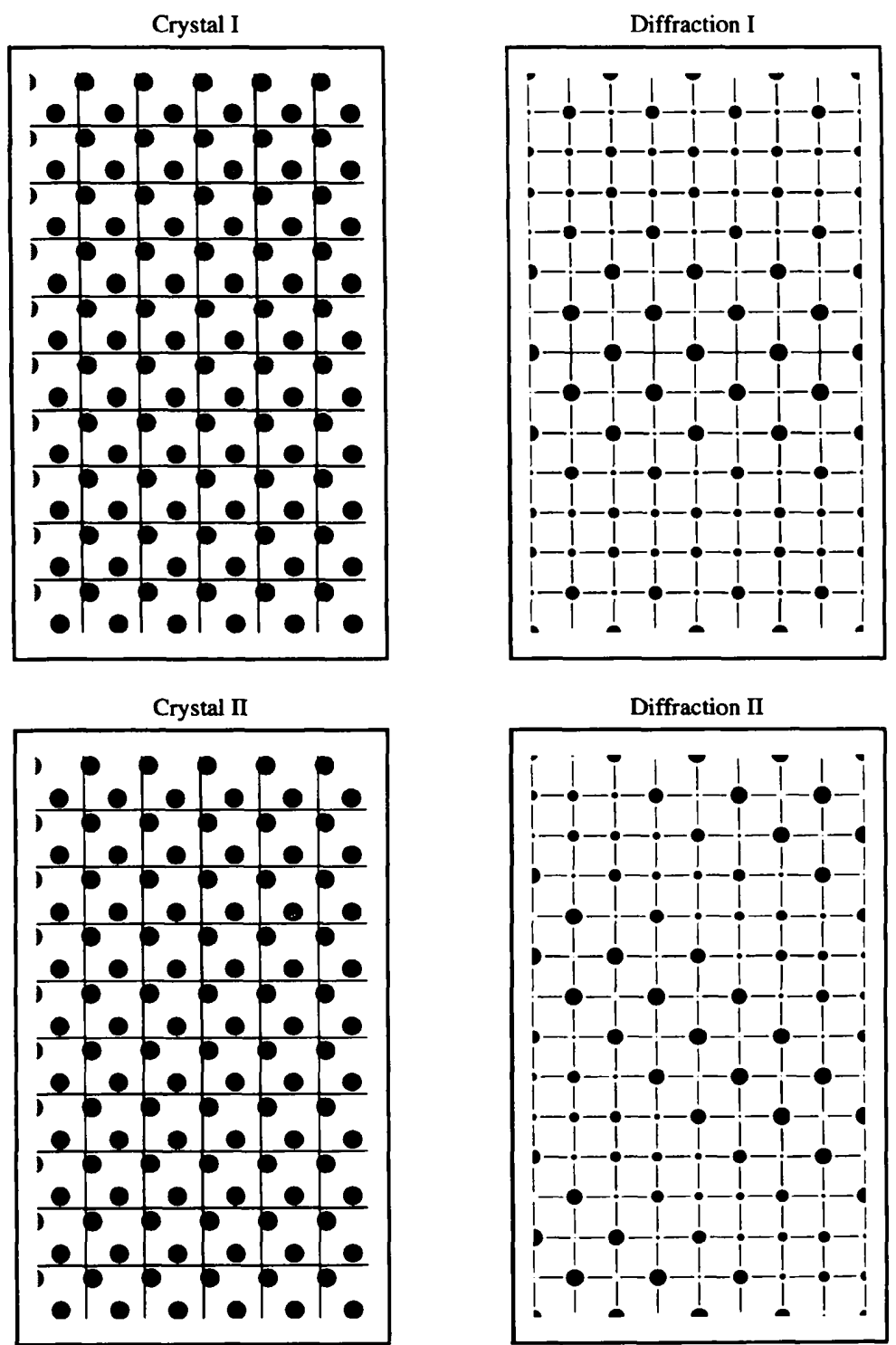

Fig. 3. An example of glide symmetry and its effect on the diffraction pattern. Crystal I is based on a square lattice with plane group $p 1 g 1$ (one of the atomic positions in the unit cell is obtained by applying a horizontal glide transformation to the other). The resulting diffraction pattern (diffraction I) shows systematic absences along the horizontal axis only. The second crystal structure is included to illustrate the sensitivity of the diffraction pattern to small changes in the atomic coordinates in this structure: the second atomic position is misplaced by about $1 / 20$ of the lattice parameter, breaking the glide symmetry. 
would be such that nodes in the lattice occurred only at those points showing intense scattering in Fig. 2.

To illustrate the role of the atomic basis in determining crystal symmetry and the intensity in the associated diffraction pattern, one of the atoms can be changed to a different type. This is shown in the lower two panels of Fig. 2. In this case, of course, systematic absences will no longer occur: the rectangular cell is now primitive, so some diffracted intensity will be observed at each node of a rectangular reciprocal lattice.

\section{Glide symmetry}

Systematic absences will also arise because of glide symmetry, as shown in Fig. 3. The upper two panels in this figure show a $p 1 g 1$ structure and its diffraction pattern. The glide axis is oriented in the horizontal direction and gives rise to the systematic absences seen only along the horizontal axis of the diffraction pattern.

To show the sensitivity of the diffraction pattern to the atomic positions, one of the atoms has been displaced by about $1 / 20$ of the lattice constant along the horizontal direction (lower panels in Fig. 3). This lifts the systematic absence conditions of the glide and causes quite noticeable changes to the diffraction pattern, although the changes to the structure are barely visible.

These structures also provide an illustration of Friedel's law. Neither crystal possesses a center of inversion symmetry; however, the diffraction patterns certainly do. For this reason, in the upper right of Fig. 3, one cannot determine whether the crystal plane group is $p 1 g 1$ or $p 2 m g$ by inspecting the diffraction pattern. In the same way, the plane group in the lower figure might be $p 1$ or $p 2$, based on the diffraction data alone.

\section{Discussion}

Two-dimensional crystallography is pedagogically valuable for introducing a range of concepts. The fact that structures can be represented completely (i.e. without projection) on a screen, or paper, makes it much easier to grasp the nature of the relationships being presented. The use of computer simulation gives an immediate display of user-defined structures which reinforces an understanding and intuition for relationships between direct and reciprocal space. It also provides immediate feedback which can usefully challenge any misconceptions that may arise.

To motivate study in two dimensions and to provide a link with real three-dimensional structures, exercises based on electron diffraction analysis of structure can be used. Because of the small curvature of the Ewald sphere, electron diffraction patterns are essentially plane sections passing through the origin in reciprocal space (Vainshtein, 1994): the observed diffraction pattern can be associated, in real space, with a twodimensional structure obtained by projection of the crystal structure along the viewing axis (Cowley, 1984).

Exercises can easily be developed along these lines. For example, one can provide students with a (physical) threedimensional model of a crystal structure and a two-dimensional diffraction pattern and ask what the orientation of the crystal was when the diffraction pattern was taken. Alternatively, one can provide several two-dimensional diffraction patterns, together with the relative orientations, and ask what models of structure could give rise to these observations.
The program used in this work has evolved over recent years while teaching elementary crystallography to undergraduate students of Condensed Matter Physics and Materials Science. It has proved both useful and stimulating for students to use. Depending on class level, different degrees of sophistication are employed: from illustrating fundamental concepts like crystal lattices, spatial periodicity and the reciprocal lattice, through to the more advanced concepts presented in this article.

\section{Program overview}

The program is written in the cT programming language (Sherwood \& Andersen, 1993). (Information about the cT programming language can also be found on the www at http:// cil.andrew.cmu.edu/ct.html.) This choice was a convenient one because of the cross-platform compatibility of this programming language.

Program design has focused on developing an interactive tool for investigation of the real-space/reciprocal-space relationships for an arbitrary crystal structure. It is therefore a specialized calculation and display tool, not a guided tutorial program. It is easy to use and help screens are available throughout to provide background information and guidance.

\section{A2. User input}

The program requires a crystal structure to be defined in real space. Lattice vectors are entered in polar coordinates and the atomic basis is then described using fractional coordinates of the lattice vectors. A relative form factor can be specified for distinct atom types. Any of the 17 plane groups can be selected which applies symmetry operations, generating further atomic positions.

\section{A3. Program displays}

A variety of display modes are available for inspection of real and reciprocal space. In each case, a PostScript file can be produced for hard copy. Zooming is provided, with independent scale factors for the real and reciprocal displays. A screen buffer may be used to store a display allowing quick comparison between structures.

\section{A4. Program availability}

A copy of the program can be obtained from the authors without charge. Two versions exist: one for Macintosh and one for Windows. A PostScript-compatible printer is required for hard copy.

\section{References}

Cowley, J. M. (1984). Diffraction Physics, pp. 114-116. Amsterdam: North-Holland.

Giacovazzo, C. (1992a). Fundamentals of Crystallography, ch. 1, p. 18. Oxford University Press.

Giacovazzo, C. (1992b). Fundamentals of Crystallography, ch. 1, pp. 45-53. Oxford University Press.

International Tables for Crystallography (1983). Vol. A, edited by T. Hahn, pp. 82-99. Dordrecht: D. Reidel Publishing Co.

Sherwood, B. A. \& Andersen, D. M. (1993). Computers in Physics, 7. 136-143.

Vainshtein, B. K. (1994). Fundamentals of Crystals: Symmetry and Methods of Structural Crystallography, pp. 338-340. Berlin: Springer-Verlag. 